\title{
BMJ Open Improving early diagnosis of cardiovascular disease in patients with type 2 diabetes and COPD: protocol of the RED-CVD cluster randomised diagnostic trial
}

\author{
Amy Groenewegen (D) , ${ }^{1}$ Victor W Zwartkruis (D) , ${ }^{2}$ Michiel Rienstra, ${ }^{2}$ \\ Monika Hollander, ${ }^{1}$ Hendrik Koffijberg, ${ }^{1}$ Maarten Jan Maria Cramer, ${ }^{3}$ \\ Yvonne T van der Schouw, ${ }^{1}$ Arno W Hoes, ${ }^{4}$ Rudolf A de Boer, ${ }^{2}$ Frans H Rutten ${ }^{1}$
}

To cite: Groenewegen A, Zwartkruis VW, Rienstra M, et al. Improving early diagnosis of cardiovascular disease in patients with type 2 diabetes and COPD: protocol of the RED-CVD cluster randomised diagnostic trial. BMJ Open 2021;11:e046330. doi:10.1136/ bmjopen-2020-046330

- Prepublication history for this paper is available online. To view these files, please visit the journal online (http://dx.doi. org/10.1136/bmjopen-2020046330).

Received 08 November 2020 Accepted 01 October 2021

D) Check for updates

(C) Author(s) (or their employer(s)) 2021. Re-use permitted under CC BY-NC. No commercial re-use. See rights and permissions. Published by BMJ.

For numbered affiliations see end of article.

Correspondence to Amy Groenewegen; a.groenewegen-11@ umcutrecht.nl

\section{ABSTRACT}

Introduction The early stages of chronic progressive cardiovascular disease (CVD) generally cause non-specific symptoms that patients often do not spontaneously mention to their general practitioner, and are therefore easily missed. A proactive diagnostic strategy has the potential to uncover these frequently missed early stages, creating an opportunity for earlier intervention. This is of particular importance for chronic progressive CVDs with evidence-based therapies known to improve prognosis, such as ischaemic heart disease, atrial fibrillation and heart failure.

Patients with type 2 diabetes or chronic obstructive pulmonary disease (COPD) are at particularly high risk of developing CVD. In the current study, we will demonstrate the feasibility and effectiveness of screening these highrisk patients with our early diagnosis strategy, using tools that are readily available in primary care, such as symptom questionnaires (to be filled out by the patients themselves), natriuretic peptide measurement and electrocardiography.

Methods and analysis The Reviving the Early DiagnosisCVD trial is a multicentre, cluster randomised diagnostic trial performed in primary care practices across the Netherlands. We aim to include $1300(2 \times 650)$ patients who participate in a primary care disease management programme for COPD or type 2 diabetes. Practices will be randomised to the intervention arm (performing the early diagnosis strategy during the routine visits that are part of the disease management programmes) or the control arm (care as usual). The main outcome is the number of newly detected cases with CVDs in both arms, and the subsequent therapies they received. Secondary endpoints include quality of life, cost-effectiveness and the added diagnostic value of family and reproductive history questionnaires and three (novel) biomarkers (highsensitive troponin-I, growth differentiation factor-15 and suppressor of tumourigenicity 2). Finally newly initiated treatments will be compared in both groups.

Ethics and dissemination The protocol was approved by the Medical Ethical Committee of the University Medical Center Utrecht, the Netherlands. Results are expected in

\section{Strengths and limitations of this study}

- This is the first randomised controlled diagnostic trial to evaluate a symptom-focused intervention for detection of three cardiovascular diseases that can be readily implemented by trained nurses in routine primary care for patients with type 2 diabetes or chronic obstructive pulmonary disease.

- Patient-relevant outcomes, such as new-onset ischaemic heart disease, atrial fibrillation and heart failure, and quality of life, will be evaluated, and the treatment initiated.

- A potential limitation is the presence of cardiovascular risk disease management programmes in many Dutch primary care practices that already aim to improve the risk profiles of community-dwelling adults and may, therefore, limit the contrast between our intervention and control arms.

- Providing patients and general practitioners (GPS) with selective information depending on the randomisation result will prevent GPs in the control arm from performing (parts) of the intervention, but may lead to differences in participation rate and baseline characteristics between groups.

2022 and will be disseminated through international peerreviewed publications.

Trial registration number NTR7360.

\section{INTRODUCTION}

Diagnosing non-acute cardiovascular diseases (CVDs) in an early stage may be difficult because symptoms can be non-specific or atypical. This seems even more true in patients with chronic obstructive pulmonary disease (COPD) or type 2 diabetes mellitus (T2DM) because of similarities in signs and symptoms (eg, shortness of breath and reduced exercise tolerance). Although it is well known that patients with COPD and T2DM are at 
increased risk of developing $\mathrm{CVD},{ }^{1-4}$ a change in symptoms hinting at a new CVD may remain unnoticed by both patient and physician. Selective screening studies in patients with COPD or T2DM aged over 60-65 years showed high prevalence of unrecognised heart failure (HF); $21 \%$ and $28 \%$, respectively, ${ }^{56}$ while unrecognised coronary artery disease may even be observed in up to $65 \%$ of patients with T2DM. ${ }^{7}$

In the Netherlands, most patients with COPD or T2DM are managed in primary care disease management programmes by general practitioners (GPs) and trained practice nurses. Little attention is paid to proactive screening and recognition of CVD within these disease management programmes, although it is well established that CVDs contribute significantly to morbidity and mortality in both COPD ${ }^{89}$ and T2DM. ${ }^{10-12}$ A proactive diagnostic strategy could thus be useful for early CVD detection, before a critical acute event (eg, acute myocardial infarction, stroke or acute HF) occurs. Such an early diagnosis strategy could easily be blended with the existing disease management programmes and may improve the care of these high-risk patients in a costeffective way. The strategy we propose will focus on early detection of coronary artery disease, atrial fibrillation and HF because of the availability of well-established mortality and morbidity reducing treatments, which are also effective in early stages of these CVDs, with the only exception nowadays for HF with preserved ejection fraction.

Our 'Early Diagnosis Strategy' comprises two stages. In the first stage, patients will fill out a questionnaire on demographic characteristics and signs and symptoms suggestive of CVD. Patients with a score indicating high risk of CVD will proceed to the second stage and will undergo a focused physical examination, electrocardiography and natriuretic peptide measurements. If test findings indicate presence of underlying CVD, the patient will be referred to a cardiologist for further investigations and treatment will be initiated if a novel cardiac diagnosis is established.

In this paper, we describe the study design of the Reviving the Early Diagnosis of CVD (RED-CVD) cluster randomised diagnostic trial, which will investigate the yield of this early diagnosis strategy compared with usual care in terms of newly recognised CVD. In addition, changes in treatment, the effect on health-related quality of life (HR-QoL) and the cost-effectiveness of the strategy will be evaluated. Finally, we plan to evaluate the incremental value of several (novel) biomarkers and a detailed questionnaire on family-specific and female-specific history in diagnosing CVD.

\section{Objectives}

Our primary objective is to determine the diagnostic yield of the early diagnosis strategy compared with usual care, in terms of detection of previously unrecognised coronary heart disease, atrial fibrillation and $\mathrm{HF}$ in patients with COPD or T2DM who participate in primary care disease management programmes.
Secondary objectives are to assess the effect of the early diagnosis strategy on HR-QOL and the cost-effectiveness of the early diagnosis strategy compared with usual care.

In additional exploratory analyses, we will evaluate the diagnostic value of family-specific and female-specific history taking and the diagnostic value of contemporary biomarkers.

\section{METHODS}

\section{Study design}

We are currently conducting a cluster randomised diagnostic trial with general practices as the unit of randomisation. The first participant was included on 19 June 2019 and the trial is projected to run until 1 December 2022. During 1 year of follow-up, the numbers of newly diagnosed CVD, the subsequent treatments and the treatment effects in terms of HR-QoL will be compared between the intervention and control arms. Furthermore, the cost-effectiveness of the early diagnosis strategy will be assessed. Within the intervention arm, the diagnostic value of the family-specific and female-specific questionnaires as well as three cardiac biomarkers will be evaluated (see figure 1).

\section{Study population, recruitment and randomisation}

General practices across the Netherlands will be recruited over the course of 2 years. Before randomisation, GPs and practice nurses receive general information on the objective and purpose of the study, but no detailed information on the actual intervention. All general practices with active disease management programmes for COPD and/ or diabetes will be eligible. During the recruitment phase, practices will be randomly allocated to either the early diagnostic strategy or usual care. We are using cluster randomisation instead of randomisation at an individual participant level to avoid contamination of participants and GPs between the groups. A computer-based block randomisation with a 1:1 allocation ratio will be performed based on general practice size, to avoid large imbalances in numbers of participants between both arms of the trial. ${ }^{13}$ If, during subsequent randomisation, there appears to be an unequal distribution of patients across both arms (eg, due to clustering effects or due to the informed consent procedure, in which patients receive selective information based on the randomisation of their general practice, also see below), the randomisation module will be adapted to a 2:1 allocation ratio.

Patients who participate in a primary care disease management programme for COPD or T2DM will be selected from the electronic medical files of participating GPs. In the Netherlands, all residents are registered with a GP, who has a gatekeeper's position. Generally, patients only have access to specialist care after consultation with their GP. Of all patients with T2DM in the Netherlands, $88 \%$ participate in a primary care disease management programme for T2DM. 


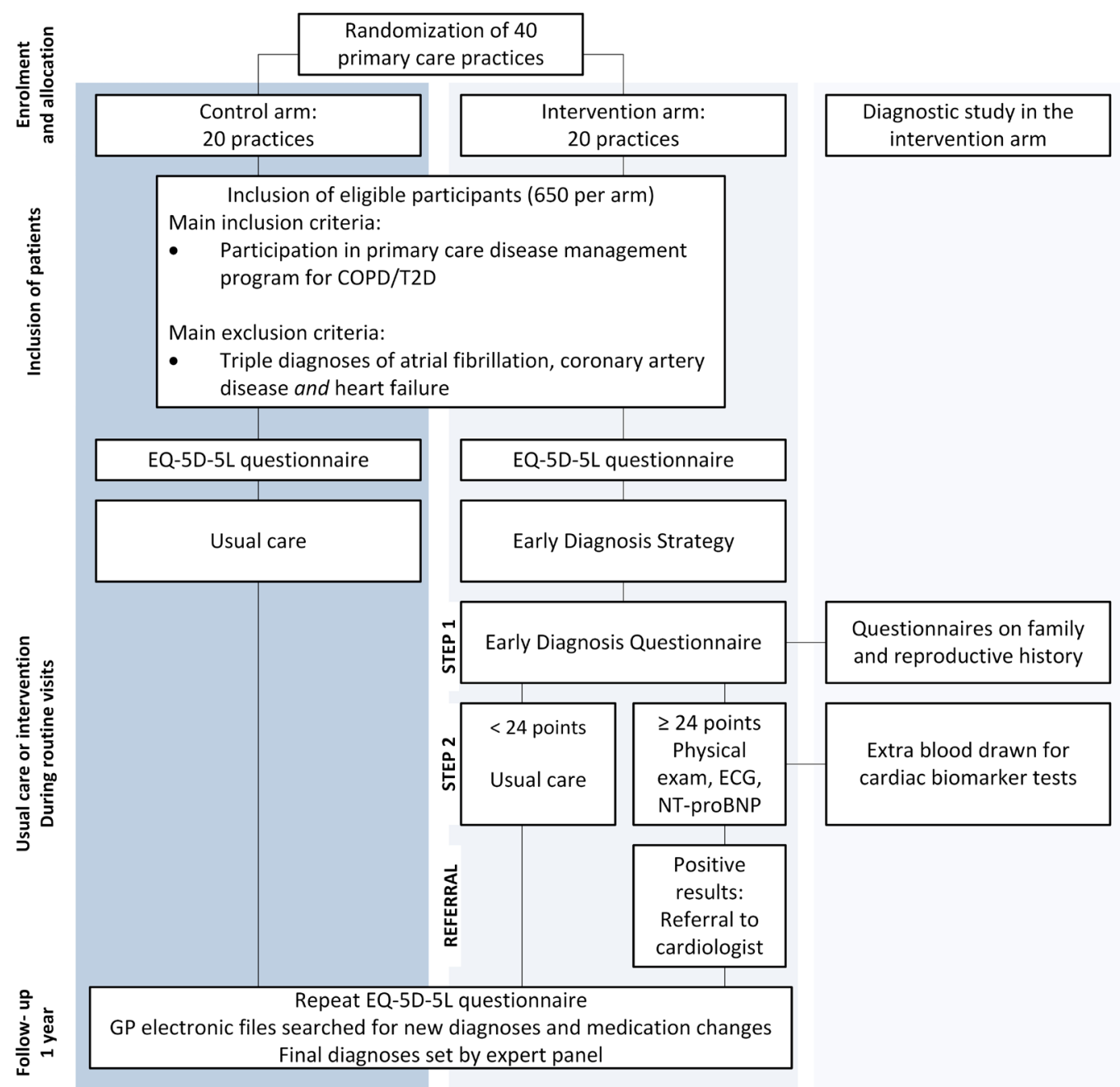

Figure 1 Study procedures. COPD, chronic obstructive pulmonary disease; GP, general practitioner; NT-pro-BNP, N-terminal pro b-type natriuretic peptide; T2D, type 2 diabetes.

Patients with an established triple diagnosis of coronary artery disease and $\mathrm{HF}$ and atrial fibrillation will be excluded. These baseline diagnoses will be considered present only when documented with echocardiography in case of HF, coronary angiography, stress tests, coronary CT-A or an Agatston calcium score $>100$ on CT scan in case of coronary heart disease, and with electrocardiography (or a single lead-ECG of at least $1 \mathrm{~min}$, Holter or loop recording) in the case of atrial fibrillation. Patients with one or two established CV target diagnoses will not be excluded, because they may still have developed another CVD, which may be uncovered by the early diagnosis strategy.

\section{Sample size calculations}

Based on the prevalence in previous screening studies of unrecognised HF $(21 \%-28 \%)$ and coronary artery disease $(65 \%)$ in patients with COPD or T2DM, and the prevalence of unrecognised atrial fibrillation $(1.4 \%)$ in the elderly population, ${ }^{14}$ we estimate to uncover these target CVDs in at least $10 \%$ of the patients with the early diagnosis strategy, compared with $5 \%$ with usual care. To detect this $5 \%$ difference in new diagnoses, using an alpha of 0.05 , a power of 0.80 , an intracluster correlation coefficient of $0.01^{15-17}$ and an anticipated drop-out of $10 \%, 650$ participants per arm will be required. In order to recruit 1300 participants within a year, we estimate that participation of 40 general practices is needed, as approximately 150 patients participate in a disease management programme for COPD or T2DM per (average-sized) general practice per year.

\section{Blinding}

The study will be conducted in a single blind manner. GPs will be asked to participate in a study investigating the early detection of CVD in high-risk patients with COPD or T2DM. Participating GPs will only receive more detailed information after their practice has been randomised. The design of early diagnosis strategy will be disclosed to GPs in the usual care arm after completion of the study. Selectively informing the GPs about the intervention will prevent those in the control group from applying (components of) the early diagnosis strategy. Patients in the control group will be asked to participate in a study on the occurrence of CVDs and their impact on quality of life, and will be informed on the existence of 
an intervention arm after the study has been completed, to prevent priming of participants. The adjudication panel responsible for setting the final diagnoses will be blinded for allocation to intervention or usual care. The panel will, however, receive information on the results of components of the early diagnosis strategy (ECG, $\mathrm{N}$-terminal pro b-type natriuretic peptide, NT-pro-BNP) because these are essential for accurate diagnosing. This may introduce some incorporation bias, but setting a diagnosis as accurately as possible has higher priority.

\section{Diagnostic intervention study}

The early diagnosis strategy will be implemented in the existing primary care disease management programmes. No additional patient contacts are necessary, as all study procedures will take place during the routine consultations of the disease management programmes.

Participating GPs will send a letter to all eligible patients. A prescreening log counting all eligible and ineligible patients will be kept. Those who are interested in participating will receive further information and will be asked for informed consent by the researcher before any study procedure is undertaken. Before their next routine practice visit, patients in both arms will fill out the EQ-5D-5L questionnaire about HR-QoL ${ }^{18} 19$ at home. In addition, patients in the intervention arm will fill out the Early Diagnosis Questionnaire and family questionnaire. Women will also fill out the reproduction questionnaire. During the routine visit, the practice nurse will apply step 2 of the early diagnosis strategy (physical examination, ECG and NT-pro-BNP measurement) in patients whose questionnaires indicate (increased risk of) CVD. Participants in the control arm will receive usual care. After 1 year of follow-up, medical files of all participants will be reviewed for documented new $\mathrm{CV}$ diagnoses, changes in CV medication status, CVD-related hospitalisations and use of the primary care facility during the past year. Additionally, they will be asked to fill out the EQ-5D-5L questionnaire again.

\section{The Early Diagnosis Questionnaire}

As described above, the early diagnosis strategy is a two-stage intervention. The first step comprises a questionnaire on demographic characteristics, and signs or symptoms suggestive of one of the three target CVDs (see table 1). The Early Diagnosis Questionnaire was developed using data from the Lifelines cohort study, which includes information on patient characteristics, signs, symptoms, self-reported diseases, electrocardiography, lung function and laboratory tests of 167000 inhabitants of the northern region of the Netherlands. ${ }^{20}$ The questions included in the questionnaire, as well as the corresponding scoring model and cut-off values, were based on analyses of the LifeLines data, and are reported in more detail elsewhere. ${ }^{21}$

The early diagnosis strategy in the intervention arm

The Early Diagnosis Questionnaire will be filled out at home by the participants in the intervention arm, prior to the routine visit scheduled for the COPD or T2DM primary care disease management programmes. The GP or the practice nurse will discuss the answers with the

Table 1 Early Diagnosis Questionnaire (translated from Dutch) and scoring model for eligibility in intervention arm

\begin{tabular}{|c|c|c|}
\hline \multirow[b]{2}{*}{ Demographic variables } & \multicolumn{2}{|c|}{ Score (for positive answers) } \\
\hline & COPD & T2DM \\
\hline 1. Age per year, starting at 40 & 1 & 1 \\
\hline 3. $\mathrm{BMI} \geq 30 \mathrm{~kg} / \mathrm{m}^{2}$ & 3 & 3 \\
\hline 4. Current smoking & 5 & 5 \\
\hline $\begin{array}{l}\text { 1. In the past } 3 \text { months, have you experienced palpitations or an unpleasant pounding of the } \\
\text { heart? }\end{array}$ & 12 & 12 \\
\hline $\begin{array}{l}\text { 2. In the past } 3 \text { months, have you experienced any chest pain or discomfort while } \\
\text { exercising? (eg, while hurrying or walking slightly uphill or bicycling) }\end{array}$ & 4 & 4 \\
\hline $\begin{array}{l}\text { 3. In the past } 3 \text { months, were you troubled by shortness of breath while exercising? (eg, } \\
\text { while hurrying or walking slightly uphill or bicycling) }\end{array}$ & NA & 4 \\
\hline 6. Do you expect your health to get worse in the coming year? & 3 & 3 \\
\hline $\begin{array}{l}\text { 7. In the past } 3 \text { months, have you experienced pain or a heavy feeling in your legs that } \\
\text { occurred while walking and disappeared at rest? }\end{array}$ & 2 & 2 \\
\hline
\end{tabular}

Breathlessness during exercise (question 3 ) had insufficient discriminatory value in patients with COPD and was therefore considered not applicable (NA) in COPD participants in the intervention arm.

BMI, body mass index; COPD, chronic obstructive pulmonary disease; NA, not applicable; T2DM, type 2 diabetes mellitus. 
participants. All participants with a score $\geq 24$ (see table 1) will proceed to the second stage of the intervention and will undergo a focused physical examination, ECG and blood measurement of NT-pro-BNP.

The physical examination includes auscultation of heart and lungs, palpation of the apical beat and radial pulse, and inspection of the legs. Physical examinations will be performed by either a trained practice nurse or the GP, in a standardised manner. Signs of fluid overload (ankle oedema, pulmonary crepitation), irregular pulse or tachycardia (>100 bpm in rest), abnormal heart murmurs, or a broadened/sustained apical beat in left decubital position will be considered abnormal. ${ }^{522} 23$ A standard 12-lead ECG will be recorded by a trained employee of the GP or a primary care laboratory and will be interpreted by an experienced GP or cardiologist. ECG findings considered abnormal include rhythm disturbances (atrial fibrillation or atrial flutter), left axis deviation, serious conduction abnormalities (second and third degree atrioventricular block (AV-block), complete left bundle branch block, trifascicular block), pathological Q-waves, voltage criteria for left ventricular hypertrophy, T-wave abnormalities and ST-deviations. Venous blood samples will be taken and circulating NT-pro-BNP levels will be determined. An NT-pro-BNP $\geq 125 \mathrm{ng} / \mathrm{L}$ will be considered abnormal as recommended in the 2016 European Society of Cardiology (ESC) guidelines on HF. ${ }^{24}$

In case of one or more abnormal findings in the second stage of the intervention (ie, prespecified ECG abnormalities, elevated NT-pro-BNP and/or signs suggestive of CVD on physical examination), the GP is asked to refer to a cardiologist for additional investigations, for example, echocardiography, Holter monitoring and/or imaging of the coronary arteries to come to a final diagnosis.

In addition, in the patients in the intervention arm who undergo blood sampling for NT-pro-BNP measurement, an extra $10 \mathrm{~mL}$ blood sample will be drawn during the same venepuncture. After centrifugation, EDTA plasma and serum will be aliquoted and stored at $-80^{\circ} \mathrm{C}$. At the end of the follow-up period, serum samples will be thawed and levels of three cardiac biomarkers (growth differentiation factor-15 (GDF-15), suppressor of tumourigenicity 2 (ST-2) and high sensitive troponin I (hs-Tn I) ) will be determined. Remaining samples are stored in a biobank to enable future biomarker studies.

\section{Control arm: usual care}

Patients in the control arm will receive usual care in the disease management programmes for COPD or T2DM. It is not common practice to proactively ask patients for symptoms but if patients spontaneously complain of symptoms such as mentioned in table 1, the GP will provide usual care likely including further CV investigations and/or referral.

\section{Outcome measures}

Newly diagnosed HF, atrial fibrillation and coronary artery disease The composite outcome of new cases of the three types of target CVDs will be determined up to 1 year after the baseline visit. Using an approach similar to the prospective, randomised, open-label, blinded end-point design, the final diagnoses are set by a joint panel consisting of three members (either two cardiologists and one GP or three cardiologists) who will evaluate the results of all diagnostic tests that took place during the follow-up period. Adjudication of diagnoses by a panel of physicians is an accepted reference standard for the evaluation of $\mathrm{HF}$, for which a true reference standard is lacking. ${ }^{25} \mathrm{In}$ line with the 2016 ESC guideline on HF, the presence of symptoms and signs as well as echocardiographic evidence of left ventricular dysfunction (structural and/ or functional) will be necessary for establishing the diagnosis of HF. This means that HF is considered present when the participant mentioned suggestive symptoms on the Early Diagnosis Questionnaire (eg, exertional shortness of breath and/or reduced exercise tolerance) or have suggestive signs with physical examination (eg, broadened/sustained apical beat, pulmonary crepitation, ankle oedema) in combination with echocardiographic evidence of LV dysfunction at rest. In participants who are already on treatment with (loop) diuretics, signs of volume overload may be masked, and therefore in such patients signs of volume overload are not obligatory. ${ }^{24}$

For coronary artery disease and atrial fibrillation, widely accepted criterion standards exist and will be applied by the panel. For the diagnosis of coronary artery disease, a positive stress test (eg, ST-deviation on an exercise test or a positive (dobutamine) stress echocardiography, stress single photon-emission computed tomography (stress SPECT), stress MRI or stress positron emission tomography (stress PET)) or any imaging modality showing anatomical proof for coronary heart disease (coronary angiography, coronary CT-A or a calcium Agatston score $>100$ with CT-scan) is a prerequisite. ${ }^{26}$ For the diagnosis of (paroxysmal) atrial fibrillation, rhythm documentation is required using a standard 12-lead ECG (or a single lead-ECG of at least $1 \mathrm{~min}$, or a Holter or loop recording) showing the typical pattern of atrial fibrillation: completely irregular RR intervals and no discernible, distinct $\mathrm{P}$ waves. ${ }^{27}$

During the year of follow-up, acute forms of heart disease may occur. These will be included in the analyses if they can be categorised as acute coronary heart disease (eg, acute myocardial infarction) or acute HF or acute atrial fibrillation. In case of hospitalisation for a cerebrovascular accident, information on the presence or absence of atrial fibrillation recorded with telemetry will be requested from the hospital in question.

In case the panel does not reach consensus, a majority decision will count. There will be a random sample retesting of $10 \%$ by the same panel, blinded to the outcome, to evaluate the reproducibility of the panel diagnoses.

Health-related quality of life

HR-QoL will be measured with the EQ-5D-5L questionnaire. The EQ-5D-5L comprises five dimensions 
(mobility, self-care, usual activities, pain/discomfort and anxiety/depression), which are divided into five degrees of severity, ranging from 'no problems' to 'extreme problems. In addition, the patient's self-rated health is recorded on a Visual Analogue Scale. The Dutch tariff will be used to generate index scores (utility values) based on EQ-5D-5L outcomes. ${ }^{19}$

\section{Costs}

Care volumes regarding diagnostic investigations, outpatient visits, CV hospitalisations and prescribed medication will be collected from the GP's electronic medical files for patients in both arms. Additionally, costs of performing the early diagnosis strategy in the intervention group and usual care in the control group will be calculated, including costs of treatment and treatment complications after diagnosis. Prices will be based on market prices and tariffs.

\section{Secondary endpoints: a diagnostic study within the intervention} arm

In addition the diagnostic intervention study, a diagnostic study within the intervention arm will be performed. All patients in the intervention arm will be asked to fill out questionnaires on family history of CVD in first-degree and second-degree relatives. Women will be asked to complete a questionnaire on reproductive history and pregnancy complications. The additional questionnaires will be provided together with the Early Diagnosis Questionnaire prior to the patient's routine visit, but they are not a part of the strategy and will be analysed separately against the newly detected CVDs.

At the end of the study, the added diagnostic value of hs-Tn I, GDF-15, and ST-2 will be evaluated for the clinical endpoint of presence or absence of previously undetected HF, atrial fibrillation or coronary heart disease.

The added diagnostic value of a positive family history for CVDs in first-degree or second-degree relatives will be assessed using a questionnaire. In women, the additional diagnostic value of detailed information on reproductive history (eg, early menopause, gestational hypertension or (pre-) eclampsia, gestational diabetes, miscarriages or preterm birth) will also be evaluated.

\section{Data analyses}

\section{Diagnostic intervention study}

The yield in terms of previously unrecognised CVD will be calculated as a proportion with $95 \%$ CIs in both study arms, as well as the absolute difference in yield between the arms with $95 \%$ CI. A random effects regression model will be used with clusters (practices) introduced as frailty term, to account for the clustered design. The difference in mean HR-QoL (with 95\% CI) between the intervention and control arm at baseline and after 1 year of follow-up will be compared using a linear mixed effects model, again applying the random cluster effect.

\section{Cost-effectiveness analyses}

The cost-effectiveness of the early diagnosis strategy will be evaluated in comparison with usual care and expressed in terms of costs or cost savings per additional correct diagnosis and per quality-adjusted life-year gained. For measuring direct costs, data on resource use will be collected based on observed volumes of each of the included tests, the number of consultations (with cardiologists and GPs) and additional investigations, and the volumes of preventive medication and treatment prescribed. A decision analytical model will be developed to estimate long-term consequences for which actual CVD risks from the current study will be complemented with evidence on treatment effects, CVD risks and long-term consequences of CVD events from literature. Probabilistic analyses will be performed to determine the robustness of the cost-effectiveness results. Results will be presented in incremental cost-effectiveness ratio planes and as costeffectiveness acceptability curves, separately for men and women.

Secondary endpoints: a diagnostic study within the intervention arm

In the intervention arm, detailed family history and reproductive history for women will be considered as a diagnostic variable. The value added to the early diagnosis strategy will be evaluated with multivariable logistic regression analyses for the outcome of presence or absence of coronary heart disease, atrial fibrillation and HF.

The added diagnostic information from three biomarkers (GDF-15, hs-Tn I and ST-2) will be quantified also by multivariable logistic regression analyses, with newly detected CVD during follow-up as the binary outcome of the model. Biomarkers will be added as continuous variables to a model including the results of the early diagnosis strategy.

\section{Missing values}

For the composite outcome of newly detected CVDs, information on new diagnoses will be requested from GPs, hospitals and the Dutch death registries. Therefore, very few missing values are to be expected and a complete case analysis will be performed. For the second endpoints based on questionnaires of HR-QoL, family history and female-specific history, multilevel multiple imputation will be used to estimate missing data, as that will reflect the lack of independence usually found in clusters.

\section{Patient and public involvement statement}

During a series of user committee meetings, patients were involved in designing the informed consent procedure and informed consent letters. In addition, they delivered input together with practice nurses and GPs for practicalities of the early diagnosis strategy; how to use the early diagnosis questionnaire and perform the physical examination. 


\section{ETHICS AND DISSEMINATION}

This study will be conducted according to the principles of the current version of the declaration of Helsinki and in accordance with the Dutch law on Medical Research Involving Human Subjects Act. The study was approved by the medical ethics committee of the University Medical Center Utrecht, the Netherlands. Results of the trial are expected in 2022 and will be disseminated through peerreviewed publications.

At the study's conclusion, former participants and healthcare providers will be informed of the results through local presentations and patient-friendly publications. Patients will be actively approached to discuss outcomes. If effective, this practical protocol can be easily implemented in other primary care facilities. Practices who were randomised to the control arm will be offered training and material to perform the intervention. Promotion of the intervention will be achieved through the infrastructure of the Dutch Heart Foundation, which is widely recognised for its dissemination activities.

Research data will be made publicly available to the scientific community after publication of the main results. Researchers are encouraged to apply for subanalyses in our biobank samples, and requests will be reviewed by the principal investigator and the University Medical Centre Utrecht biobank regulatory committee.

\section{DISCUSSION}

The RED-CVD trial will demonstrate if an early diagnosis strategy is effective in terms of detection of coronary artery disease, $\mathrm{HF}$ and atrial fibrillation, in primary care patients with COPD or T2DM. Comparison with usual care will reveal whether the two-staged early diagnosis strategy will result in substantially more diagnoses. Incremental costs and cost-effectiveness will be assessed. In addition, we will evaluate the added diagnostic value for diagnosing HF, coronary artery disease and atrial fibrillation of family and reproductive history taking and selected biomarkers.

If effective, subsequent targeted treatment with established therapies may result in prognostic benefits for this population regarding morbidity and HR-QoL, but this must be prospectively tested in subsequent studies.

\section{Potential limitations}

A potential limitation of this study is the modified informed consent procedure, in which patients and GPs receive selective information, based on the allocation of their general practice to intervention or control arm. This may lead to imbalances in participation rate and even baseline characteristics but was deemed necessary to prevent priming of patients and dilution of the effect of the intervention. Second, many Dutch primary care practices have $\mathrm{CV}$ risk management programmes, which aim to improve the risk profiles of community-dwelling adults and may, therefore, limit the contrast between our intervention and control arms. However, these programmes focus on risk factor modification, rather than on suspected symptoms or yet unrecognised CVD.

\section{Relation to other studies}

It is well established that CVD contributes significantly to both morbidity and mortality in COPD and T2DM. Even so, opportunistic screening studies reported that unrecognised, new-onset CVD is still highly prevalent in these high-risk individuals. ${ }^{2516232829}$ In many of these studies, a large proportion of patients did indicate having symptoms when asked pro-actively, suggesting that the early phases of CVD might often be symptomatic but overlooked, rather than asymptomatic. ${ }^{2} 2328$ Since many cardiac pathologies can be slowed but not easily reversed, waiting for patients to present themselves with clear symptoms seems a missed opportunity. GPs and practice nurses, who see patients with COPD and T2DM on a regular basis, can play a unique role in identifying individuals who show progression on the CVD continuum. Nevertheless, primary care disease management programmes focus primarily on risk factor assessment rather than on detection of early symptoms as direct markers of disease, and studies investigating doctor-initiated strategies including proactive symptom-monitoring are rare.

The second step of the intervention combines several diagnostic tests that have already proven to be effective or promising as a screening strategy in previous studies including similar populations. For example, the natriuretic peptides NT-pro-BNP and BNP are increasingly recognised as powerful tools for screening and prevention of HF. Two large randomised controlled trials (RCTs) have reported on the use of natriuretic peptides for the identification of elevated-risk patients and the implementation of CV prevention strategies. The Screening TO Prevent HF trial randomised 1374 asymptomatic, primary care patients with at least one CV risk factor (eg, hypertension, diabetes, obesity, hyperlipidaemia or prior CVD) to BNP-guided therapy versus usual care. Patients in the BNP-guided group had plasma levels measured annually and received echocardiography and specialised collaborative care with individualised uptitration of renin-angiotensin system (RAS) antagonists and beta-blockers, for BNP values $>50 \mathrm{ng} / \mathrm{L}$. After 4.2 years of follow-up, the intervention had reduced the occurrence of the primary outcome of asymptomatic left ventricular dysfunction or overt HF $(8.7 \%$ vs $5.3 \%$, OR: 0.55 ; $95 \%$ CI: 0.37 to 0.82 ; number needed to treat: 29 per 4.2 years).$^{30}$ In 300 patients with T2DM, no history of cardiac disease and NT-pro-BNP levels of $125 \mathrm{ng} / \mathrm{L}$ or higher in the PONTIAC study, uptitration of RAS-antagonists and beta-blockers in an outpatient cardiology clinic reduced the risk of hospitalisation or death due to cardiac disease by a remarkable $65 \%$ (HR: 0.35 ; $95 \%$ CI: 0.13 to 0.98 ). Of note, the event rates in the 'uptitration' arm were similar to those in patients who had NT-pro-BNP levels $<125 \mathrm{ng} / \mathrm{L}$ at the start of the trial and were not randomised but followed for CV events. ${ }^{4}$ These studies indicate that there is an important role for 
natriuretic peptides in both screen-detection and therapy guidance of $\mathrm{HF}$.

Screening for atrial fibrillation has been the subject of much recent debate. The most feared complication of atrial fibrillation is stroke, which is often fatal or debilitating yet largely preventable with anticoagulants. Stroke registries show that unknown and untreated or undertreated atrial fibrillation accounts for most of the strokes. ${ }^{31}{ }^{32}$ In patients with atrial fibrillation and coexisting $\mathrm{T}_{2} \mathrm{DM}^{33}$ or COPD, ${ }^{34}$ there is an even larger risk of stroke and other CVDs. Data from RCTs are lacking, but large cohort studies suggest that screen-detected atrial fibrillation, particularly in the presence of additional risk factors, is not harmless and often requires anticoagulant treatment. For example, in a cohort of 5555 asymptomatic patients with atrial fibrillation, detected incidentally in primary care, treatment with anticoagulants was associated with a reduced adjusted risk of stroke $(4 \%-1 \%$, adjusted HR $0.35 ; 95 \%$ CI: 0.17 to 0.71 ) and death ( $7 \%$ to $4 \%$, adjusted HR: $0.56 ; 95 \%$ CI 0.36 to 0.85 ) over the course of 1.5 years, compared with no treatment. ${ }^{35}$ Screening studies for silent atrial fibrillation in patients with T2DM and/or COPD are lacking, but opportunistic screening (using pulse palpation or ECG) seems cost-effective in elderly populations ( eg, $>65$ years ${ }^{36}$ and in other at risk populations. ${ }^{37}$ In individuals $>65$ years, screening revealed previously undetected atrial fibrillation in $1.4 \%,{ }^{14}$ corresponding to a number needed to screen of 70. Because both COPD and T2DM are independently associated with atrial fibrillation, ${ }^{3839}$ we expect the number needed to screen to be lower in this population.

Trials investigating routine screening for coronary artery disease have had mostly neutral results on major cardiac outcomes, even in high-risk individuals. ${ }^{40}$ These trials included asymptomatic patients only; trials including screening for symptoms or stepwise screening methods are lacking, even though history taking is generally regarded as a cornerstone of coronary artery disease diagnosis. For example, the large population-based Rotterdam Study demonstrated a strong association between Rose questionnaire angina pectoris and the amount of coronary calcification found with CT scanning. ${ }^{41}$ In another study including 11223 patients with stable angina referred for coronary angiography, symptoms of stable ischaemic heart disease were predictive of increased risk of adverse CV outcomes after adjusting for traditional cardiac risk factors, even in the absence of obstructive coronary artery disease. ${ }^{42}$

Nonetheless, whether (opportunistic) screening for CVD is useful remains controversial, even in populations at increased CV risk, and international guidelines on this subject vary. Potential harms include the risk of additional (invasive) diagnostic techniques and treatments, the negative consequences of being labelled 'unhealthy' and the related cost. The American College of Cardiology, therefore, recommends against screening of asymptomatic high-risk patients owing to a lack of outcome benefit, and emphasises the potential risk of procedural harms since abnormal screening results often require additional (invasive) investigations. ${ }^{43}$ Indeed, because it targets individuals who did not already select themselves as being symptomatic, proactive $\mathrm{CV}$ care is per definition less precise than reactive care and holds the risk of excessive use of cardiac follow-up tests and referrals. However, by performing our diagnostic strategy in a staged manner, the presence of symptoms or a very high-risk profile becomes a precondition for any additional testing, thereby minimising the risk that the additional tests in question are unnecessary. The recently published ESC guideline on (pre-) diabetes and CVD recommend ECG screening for atrial fibrillation in diabetes patients $>64$ years old. ${ }^{44}$ In contrast, the updated US Preventive Services Task Force recommendations cite a lack of evidence to assess the net benefits vs net harms of screening ECGs in high-risk patients. ${ }^{45}$ Thus, our study could be one of the building blocks for this much needed evidence.

\section{Author affiliations}

1Julius Center for Health Sciences and Primary Care, University Medical Center Utrecht, Utrecht, The Netherlands

${ }^{2}$ Department of Cardiology, University Medical Centre Groningen, Groningen, The Netherlands

${ }^{3}$ Department of Cardiology, University Medical Centre Utrecht, Utrecht, The Netherlands

${ }^{4}$ University Medical Centre Utrecht, Utrecht, The Netherlands

Correction notice This article has been corrected since it was published. Initials of 2 authors 'Yvonne van der Schouw' and 'Rudolf de Boer' have been updated.

Contributors FHR, RAdB, AWH, YTvdS, MJMC, HK, MH, MR and AG designed the study. FHR, MH and AG wrote the study protocol. FHR, RAdB, AWH, MH, MR, VWZ and AG developed the Early Diagnosis Questionnaire. FHR, MH, RAdB, MR, AG and VWZ included practices and acquired informed consent, VWZ and AG contributed to the implementation of the intervention. AG drafted the first version of this manuscript. All coauthors critically reviewed and revised the manuscript before providing final approval.

Funding The RED-CVD project is part of the Dutch Cardiovascular Research Programme (CVON) and received an unrestricted institutional grant (CVON2017-11) by the Dutch Heart Foundation.

Competing interests None declared.

Patient and public involvement Patients and/or the public were involved in the design, or conduct, or reporting, or dissemination plans of this research. Refer to the Methods section for further details.

Patient consent for publication Not applicable.

Provenance and peer review Not commissioned; externally peer reviewed.

Open access This is an open access article distributed in accordance with the Creative Commons Attribution Non Commercial (CC BY-NC 4.0) license, which permits others to distribute, remix, adapt, build upon this work non-commercially, and license their derivative works on different terms, provided the original work is properly cited, appropriate credit is given, any changes made indicated, and the use is non-commercial. See: http://creativecommons.org/licenses/by-nc/4.0/.

\section{ORCID iDs}

Amy Groenewegen http://orcid.org/0000-0003-2393-1927

Victor W Zwartkruis http://orcid.org/0000-0003-0115-1313

\section{REFERENCES}

1 Roversi S, Fabbri LM, Sin DD, et al. Chronic obstructive pulmonary disease and cardiac diseases. An urgent need for integrated care. Am J Respir Crit Care Med 2016;194:1319-36.

2 Boonman-de Winter LJM, Rutten FH, Cramer MJM, et al. High prevalence of previously unknown heart failure and left ventricular dysfunction in patients with type 2 diabetes. Diabetologia 2012;55:2154-62.

3 Rasmussen T, Køber L, Pedersen JH, et al. Relationship between chronic obstructive pulmonary disease and subclinical coronary 
artery disease in long-term smokers. Eur Heart $J$ Cardiovasc Imaging 2013:14:1159-66.

4 Huelsmann M, Neuhold S, Resl M, et al. PONTIAC (NT-proBNP selected prevention of cardiac events in a population of diabetic patients without a history of cardiac disease): a prospective randomized controlled trial. J Am Coll Cardiol 2013;62:1365-72.

5 Boonman-de Winter LJM, Rutten FH, Cramer MJ, et al. Efficiently screening heart failure in patients with type 2 diabetes. Eur $J$ Heart Fail 2015;17:187-95.

6 Rutten FH, Moons KGM, Cramer M-JM, et al. Recognising heart failure in elderly patients with stable chronic obstructive pulmonary disease in primary care: cross sectional diagnostic study. BMJ 2005;331:1379.

7 Muhlestein JB, Lappé DL, Lima JAC, et al. Effect of screening for coronary artery disease using $\mathrm{CT}$ angiography on mortality and cardiac events in high-risk patients with diabetes: the FACTOR-64 randomized clinical trial. JAMA 2014;312:2234-43.

8 Feary JR, Rodrigues LC, Smith CJ, et al. Prevalence of major comorbidities in subjects with COPD and incidence of myocardial infarction and stroke: a comprehensive analysis using data from primary care. Thorax 2010;65:956-62.

9 Reed RM, Eberlein M, Girgis RE, et al. Coronary artery disease is under-diagnosed and under-treated in advanced lung disease. $\mathrm{Am} J$ Med 2012;125:1228.e13-1228.e22.

10 Wingard DL, Barrett-Connor E. Heart Disease and Diabetes. In: National diabetes data group. Diabetes in America, 1995: 429-48.

11 Fox CS. Cardiovascular disease risk factors, type 2 diabetes mellitus, and the Framingham heart study. Trends Cardiovasc Med 2010;20:90-5.

12 Kannel WB, McGee DL. Diabetes and cardiovascular disease. The Framingham study. JAMA 1979;241:2035-8.

13 Scott NW, McPherson GC, Ramsay CR, et al. The method of minimization for allocation to clinical trials. A review. Control Clin Trials 2002;23:662-74.

14 Lowres N, Neubeck L, Redfern J, et al. Screening to identify unknown atrial fibrillation. A systematic review. Thromb Haemost 2013:110:213-22.

15 Smeeth L, Ng ES-W. Intraclass correlation coefficients for cluster randomized trials in primary care: data from the MRC trial of the assessment and management of older people in the community. Control Clin Trials 2002;23:409-21.

16 Fitzmaurice DA, Hobbs FDR, Jowett S, et al. Screening versus routine practice in detection of atrial fibrillation in patients aged 65 or over: cluster randomised controlled trial. BMJ 2007;335:383.

17 Uittenbogaart SB, Verbiest-van Gurp N, Erkens PMG, et al. Detecting and diagnosing atrial fibrillation (D2AF): study protocol for a cluster randomised controlled trial. Trials 2015;16:478

18 Nolan CM, Longworth L, Lord J, et al. The EQ-5D-5L health status questionnaire in COPD: validity, responsiveness and minimum important difference. Thorax 2016;71:493-500.

19 M Versteegh M, M Vermeulen K, M A A Evers S, et al. Dutch tariff for the five-level version of EQ-5D. Value Health 2016;19:343-52.

20 Scholtens S, Smidt N, Swertz MA, et al. Cohort profile: lifelines, a three-generation cohort study and Biobank. Int J Epidemiol 2015;44:1172-80.

21 Zwartkruis VW, Groenewegen A, Rutten FH, et al. Proactive screening for symptoms: a simple method to improve early detection of unrecognized cardiovascular disease in primary care. results from the lifelines cohort study. Prev Med 2020;138:106143.

22 Kelder JC, Cramer MJ, van Wijngaarden J, et al. The diagnostic value of physical examination and additional testing in primary care patients with suspected heart failure. Circulation 2011;124:2865-73.

23 Rutten FH, Cramer M-JM, Grobbee DE, et al. Unrecognized heart failure in elderly patients with stable chronic obstructive pulmonary disease. Eur Heart J 2005;26:1887-94.

24 Ponikowski P, Voors AA, Anker SD, et al. 2016 ESC Guidelines for the diagnosis and treatment of acute and chronic heart failure: The Task Force for the diagnosis and treatment of acute and chronic heart failure of the European Society of Cardiology (ESC)Developed with the special contribution of the Heart Failure Association (HFA) of the ESC. Eur Heart J 2016.

25 Bertens LCM, Broekhuizen BDL, Naaktgeboren CA, et al. Use of expert panels to define the reference standard in diagnostic research: a systematic review of published methods and reporting. PLoS Med 2013;10:e1001531.
26 Task Force Members, Montalescot G, Sechtem U, et al. 2013 ESC guidelines on the management of stable coronary artery disease: the task force on the management of stable coronary artery disease of the European Society of cardiology. Eur Heart J 2013;34:29493003.

27 Kirchhof P, Benussi S, Kotecha D, et al. 2016 ESC guidelines for the management of atrial fibrillation developed in collaboration with EACTS. Eur J Cardiothorac Surg 2016;50:e1-88.

28 van Mourik Y, Bertens LCM, Cramer MJM, et al. Unrecognized heart failure and chronic obstructive pulmonary disease (COPD) in frail elderly detected through a near-home targeted screening strategy. $J$ Am Board Fam Med 2014;27:811-21.

29 Pepine CJ, Ferdinand KC, Shaw LJ, et al. Emergence of Nonobstructive Coronary Artery Disease. J Am Coll Cardiol 2015:66:1918-33.

30 Ledwidge M, Gallagher J, Conlon C, et al. Natriuretic PeptideBased screening and collaborative care for heart failure. JAMA 2013;310:66.

31 Björck S, Palaszewski B, Friberg L, et al. Atrial fibrillation, stroke risk, and warfarin therapy revisited: a population-based study. Stroke 2013;44:3103-8.

32 Yiin GSC, Howard DPJ, Paul NLM, et al. Age-Specific incidence, outcome, cost, and projected future burden of atrial fibrillationrelated embolic vascular events: a population-based study. Circulation 2014;130:1236-44.

33 Du X, Ninomiya T, de Galan B, et al. Risks of cardiovascular events and effects of routine blood pressure lowering among patients with type 2 diabetes and atrial fibrillation: results of the advance study. Eur Heart J 2009;30:1128-35.

34 Nadeem R, Sharieff A, Tanna S, et al. Potential augmentation of the risk of ischemic cerebrovascular accident by chronic obstructive pulmonary disease in patients with atrial fibrillation. J Stroke Cerebrovasc Dis 2015;24:1893-6.

35 Martinez C, Katholing A, Freedman SB. Adverse prognosis of incidentally detected ambulatory atrial fibrillation. A cohort study. Thromb Haemost 2014;112:276-86.

36 Hobbs FDR, Fitzmaurice DA, Mant J, et al. A randomised controlled trial and cost-effectiveness study of systematic screening (targeted and total population screening) versus routine practice for the detection of atrial fibrillation in people aged 65 and over. The safe study. Health Technol Assess 2005;9:1-74.

37 Aronsson M, Svennberg E, Rosenqvist M, et al. Cost-Effectiveness of mass screening for untreated atrial fibrillation using intermittent ECG recording. Europace 2015;17:1023-9.

38 Buch $\mathrm{P}$, Friberg J, Scharling $\mathrm{H}$, et al. Reduced lung function and risk of atrial fibrillation in the Copenhagen City heart study. Eur Respir $J$ 2003;21:1012-6.

39 Pallisgaard JL, Schjerning A-M, Lindhardt TB, et al. Risk of atrial fibrillation in diabetes mellitus: a nationwide cohort study. Eur J Prev Cardiol 2016;23:621-7.

40 Clerc OF, Fuchs TA, Stehli J, et al. Non-Invasive screening for coronary artery disease in asymptomatic diabetic patients: a systematic review and meta-analysis of randomised controlled trials. Eur Heart J Cardiovasc Imaging 2018;19:838-46.

41 Oei H-HS, Vliegenthart R, Deckers JW, et al. The association of rose questionnaire angina pectoris and coronary calcification in a general population: the Rotterdam coronary calcification study. Ann Epidemiol 2004;14:431-6.

42 Jespersen L, Hvelplund A, Abildstrøm SZ, et al. Stable angina pectoris with no obstructive coronary artery disease is associated with increased risks of major adverse cardiovascular events. Eur Heart J 2012;33:734-44.

43 Wolk MJ, Bailey SR, Doherty JU, et al. ACCF/AHA/ASE/ASNC/HFSA/ HRS/SCAI/SCCT/SCMR/STS 2013 multimodality appropriate use criteria for the detection and risk assessment of stable ischemic heart disease. J Am Coll Cardiol 2014:63:380-406.

44 Cosentino F, Grant PJ, Aboyans V. 2019 ESC guidelines on diabetes, pre-diabetes, and cardiovascular diseases developed in collaboration with the EASD: the task force for diabetes, prediabetes, and cardiovascular diseases of the European Society of cardiology (ESC) and the European association for the study of diabetes (EASD). Eur Heart J 2019;41:1-69.

45 Curry SJ, Kirst AH, Owens AH, US Preventive Services Task Force. Screening for cardiovascular disease risk with electrocardiography. JAMA 2018;319:2308. 